\title{
Peripheral (Seventh) Nerve Palsy and Multiple Sclerosis: A Diagnostic Dilemma - A Case Report
}

\author{
Christian Saleh $^{a} \quad$ Olga Patsi $^{a} \quad$ Frederic Mataigne $^{b} \quad$ Stefan Beyenburg $^{a}$ \\ Departments of ${ }^{a}$ Neurology and ${ }^{b}$ Neuroradiology, Centre Hospitaleir de Luxembourg, \\ Luxembourg
}

\section{Key Words}

Multiple sclerosis · Isolated idiopathic peripheral facial palsy · Bell's palsy · Seventh nerve palsy · Peripheral demyelinating disease

\begin{abstract}
During the last decades, only a few cases on the association between peripheral demyelinating diseases and multiple sclerosis (MS) have been reported. We describe the case of a young man who was initially diagnosed with Bell's palsy, and only after performing a brain MRI was the diagnosis of MS made. We review the literature and discuss some pitfalls which may lead to missing the diagnosis of MS.

\section{Introduction}

Multiple sclerosis (MS) is a chronic autoimmune inflammatory disease characterized by demyelination and axonal degeneration of the central nervous system [1,2]. Approximately 2.5 million people are affected by MS worldwide with a 2:1 female-to-male ratio [3, 4]. MS lesions are confined to the central nervous system and consequently not extending the nerve root entry zone [5]. Isolated idiopathic peripheral facial palsy (Bell's palsy) has an annual incidence of 20-30 cases per 100,000 people per year [6]. In peripheral seventh nerve palsy, the lesion of the nerve usually occurs at the level of the geniculate ganglion (located in the facial canal) and therefore outside the central nervous system. Peripheral facial palsy, however, can also result from a central lesion at the level of the ipsilateral facial nucleus or facial nerve at the pons [6].

\section{KARGER}

Dr. Stefan Beyenburg

Department of Neurology, Centre Hospitalier de Luxembourg

4, rue Barble

LU-1210 Luxembourg (Luxembourg)

E-Mail beyenburg.stefan @chl.lu 
Saleh et al.: Peripheral (Seventh) Nerve Palsy and Multiple Sclerosis: A Diagnostic Dilemma - A Case Report

The facial nerve is primarily a motor nerve supplying the muscles of the facial expression and the stapedius muscle, but it contains also special sensory fibers to the anterior two thirds of the tongue and parasympathetic fibers to the lacrimal and salivary glands. The cortical cerebral fibers of the facial nerve descend through the posterior limb of the internal capsule to the caudal pons, where they synapse with the facial nucleus. The corticobulbar projections responsible for the innervation of the upper face are bilateral, while those for the lower facial muscles are contra- and unilateral. This particularity in neuronal connectivity explains why a unilateral supranuclear lesion, contrary to an infranuclear lesion, does not affect the muscles of the forehead and leads only to lower facial paralysis [6-8].

Over the last three decades, a few cases on the association between peripheral demyelinating diseases and MS have been reported [5, 9-11]. However, whether the association between MS and peripheral demyelinating diseases is due to different manifestations of the same underlying pathology or the expression of two distinct diseases that coincidentally occur at the same time is uncertain [5]. We describe the case of a young man who we diagnosed initially with Bell's palsy, and only after performing a brain MRI was the diagnosis of MS made.

\section{Case Report}

A 21-year-old patient presented to the emergency department complaining of brief episodes of vertigo and nausea with vomiting, which had occurred the day before. Lying still, the vertigo reduced. The initial physical examination performed in the emergency department was reported normal. There were no paresis, paresthesia, loss of balance, blurred vision, bladder problems or lack of coordination. The patient's past history was remarkable for pneumonia and a traumatic spinal fracture, from which he had fully recovered. The otorhinolaryngology examination performed on the same day of admission was normal, except for noting a slight drop of the right corner of the mouth. On the next day, the drop of the right mouth corner was prominent. He was further not able to furrow his right forehead and complained of right-sided tinnitus and numbness of the right side of his face. Closing his eyes produced the 'sign of eyelashes' (a sign of incomplete eye closure). He did not complain of nausea and vertigo. The working diagnosis of peripheral seventh nerve palsy (probably Bell's palsy) was made and steroid treatment initiated. Blood examination was normal for full blood count. Erythrocyte sedimentation rate, biochemistry, liver function, C-reactive protein, infections (including Borrelia, HIV, syphilis serologies), ANA, ANCA and antiphospholipid antibody were negative.

However, in light of the reported episodes of vertigo and nausea pointing to a brain stem lesion, we decided to perform a brain MRI that showed three demyelinating lesions in the supratentorial, periventricular region. Two lesions were on the right and one on the left side (sizes between 8-13 mm; fig. 1, fig. 2). The left periventricular lesion presented with an increase in the apparent diffusion coefficient (sign of vasogenic edema) and contrast medium enhancement. The spine MRI (fig. 3) revealed a very small lesion at the level of the right caudal pons, inferior to the facial nucleus.

Based on the brain MRI findings, a lumbar puncture was performed, which did not show any signs of inflammation. The polymerase chain reaction for EBV, CMV, HSV 1/2 and VZV was negative. Identical IgG oligoclonal bands were found in the serum and in the CSF (due to attenuation of the blood-brain barrier). Auditory evoked potentials were normal. A blink reflex was not performed. The visually evoked potentials and somatosensory evoked potentials showed a normal latency, but an amplitude reduction, which probably was due to poor 
Saleh et al.: Peripheral (Seventh) Nerve Palsy and Multiple Sclerosis: A Diagnostic Dilemma - A Case Report

cooperation. Based on the clinical and brain imaging findings, a high-dose cortisone treatment $(1,000 \mathrm{mg}$ of methylprednisolone per day for 5 days) was initiated with progressive, but slight symptom reduction.

\section{Discussion}

Isolated idiopathic peripheral facial palsy (Bell's palsy) is considered to be a disease of the peripheral nervous system, while MS is defined as a disease of the central nervous system $[1,6]$. Common MS symptoms consist in visual disturbances, paresthesia, impaired coordination and paresis [1]. These symptoms can be associated with bladder and sexual dysfunction, Lhermitte's sign (an electrical sensation along the spine by flexing the head) and ataxia. They tend to resolve spontaneously and to recur after months or even years [1].

The classical presentation of Bell's palsy is an acute or subacute unilateral facial palsy that involves the lower and the upper half of the face (contrary to a central cause, where only the lower half of the face is affected). However, it is important to keep in mind that in rare cases, peripheral facial palsy, can also 'paradoxically' be due to a central lesion at the level of the ipsilateral facial nucleus or facial nerve at the pons $[6,12]$.

The difficulty in promptly establishing the diagnosis in our patient was not due to the circumstance that he had both, i.e. peripheral and central symptoms, but that the central and peripheral symptoms did not overlap in time. While the central symptoms presented first and were transient, the following dominant and lasting symptoms were the peripheral ones, which furthermore matched perfectly the classical picture of peripheral seventh nerve palsy. The central symptomatology did not further raise the suspicion of MS. Typical MS features were absent (e.g. blurred vision, paresthesia, paresis, and time/space symptom dissemination). The slight drop of the right mouth corner in retrospect could have already been interpreted as a pointer to a central cause; however, it was so small that it was missed on two out of the three initial examinations on the day of admission. The drop of the right corner of the mouth became fully evident after $12 \mathrm{~h}$ of admission. In light of its rapid progression to a complete paralysis, it was considered to be part of the clinical picture of Bell's palsy. This is in concordance with the Bell's palsy guideline development group (GDG) definition for idiopathic peripheral nerve palsy, namely that a paresis/paralysis progresses to its maximum severity within $72 \mathrm{~h}$ of onset [13]. Other causes of peripheral seventh nerve palsy tend to develop more slowly.

It is debatable whether our patient had a distinct peripheral seventh nerve palsy, which led to a coincidental diagnosis of MS, or whether the seventh nerve palsy represented the expression of the same underlying immune-pathological dysfunction of MS [5, 14]. Uzawa and colleagues [15] reported that brain stem involvement in MS is common, but isolated peripheral nerve palsies are rare findings in MS, concluding that MS may be a cause of isolated cranial nerve palsies.

A review of the literature shows that over the last three decades, few case reports on the rare association between peripheral seventh nerve palsy and MS have been reported. Kwon and colleagues [5] described the case of a 36-year-old woman who presented initially with vertigo and right facial palsy. The patient complained further of diplopia and left-sided weakness. Initial brain MRI T2-weighted images showed a lesion at the level of the right cerebellopontine angle with contrast enhancement. Serial MRI demonstrated spatially disseminated demyelinating lesions, and nerve conduction studies were consistent with multifocal peripheral demyelinating neuropathies. Agarwal and colleagues [16] reported unilateral left-sided facial palsy, nausea, vomiting and slurred speech in a 47 -year-old male pa- 
Saleh et al.: Peripheral (Seventh) Nerve Palsy and Multiple Sclerosis: A Diagnostic Dilemma - A Case Report

tient. Medical history was positive for hypertension. His brain MRI showed a small infarct $(4 \mathrm{~mm})$ at the level of the left dorsal pons. The infarct was due to a paradoxical embolization secondary to an atrial septal defect with bidirectional shunt. Critchley [9] presented a case of a 35-year-old woman with a right-sided facial palsy. Neurological examination was normal except for a slight loss of coordination in her right upper extremity. Brain MRI and further diagnostic work-up confirmed MS. Fukazawa and colleagues [10] reported facial palsy as first symptom of MS in 4.7\% of 107 patients with MS. Ivankovic and Demarin [11] described a 35-year-old woman initially misdiagnosed with Bell's palsy. MS diagnosis was established only subsequently with the manifestation of other neurologic symptoms. Brain MRI showed disseminated supra- and infratentorial white matter lesions, which included also lesions at the level of the pons.

\section{Conclusion}

Although the GDG group recommends that clinicians should not routinely perform diagnostic imaging for patients with new-onset Bell's palsy, except when there are some evident clinical findings that would warrant imaging [13], performing a brain MRI may well be necessary in all cases of peripheral-appearing nerve palsies. Our case underlines how easily transient and brief symptoms of MS can be overlooked [1] or misinterpreted and MS diagnosis and treatment delayed, with devastating effects for the patient.

\section{Statement of Ethics}

The authors have no ethical conflicts to declare.

\section{Disclosure Statement}

The authors have no conflicts of interest to declare.

\section{References}

1 Calabresi PA: Diagnosis and management of multiple sclerosis. Am Fam Physician 2004;70:1935-1944.

2 Goldenberg MM: Multiple sclerosis review. P T 2012;37:175-184.

3 Koch-Henriksen N, Sorensen PS: The changing demographic pattern of multiple sclerosis epidemiology. Lancet Neurol 2010;9:520-532.

4 Tullman MJ: Overview of the epidemiology, diagnosis, and disease progression associated with multiple sclerosis. Am J Manag Care 2013;19(2 suppl):S15-S20.

5 Kwon JY, Kim JY, Jeong JH, Park KD: Multiple sclerosis and peripheral multifocal demyelinating neuropathies occurring in a same patient. J Clin Neurol 2008;4:51-57.

6 Gilden DH: Clinical practice. Bell's palsy. N Engl J Med 2004;351:1323-1331.

7 Tiemstra JD, Khatkhate N: Bell's palsy: diagnosis and management. Am Fam Physician 2007;76:997-1002.

-8 Gupta S, Mends F, Hagiwara M, Fatterpekar G, Roehm PC: Imaging the facial nerve: a contemporary review. Radiol Res Pract 2013;2013:248039.

-9 Critchley EP: Multiple sclerosis initially presenting as facial palsy. Aviat Space Environ Med 2004;75:10011004.

10 Fukazawa T, Moriwaka F, Hamada K, Hamada T, Tashiro K: Facial palsy in multiple sclerosis. J Neurol 1997;244:631-633.

11 Ivankovic M, Demarin V: From recurrent peripheral facial palsy to multiple sclerosis. Acta Clin Croat 2011;50:419-421. 


\section{Case Reports in Neurology}

\begin{tabular}{l|l}
\hline \multicolumn{3}{l}{ Case Rep Neurol 2016:8:27-33 } \\
\hline DOI: 10.1159/000443681 & $\begin{array}{l}\text { (c) 2016 The Author(s). Published by S. Karger AG, Basel } \\
\text { www.karger.com/crn }\end{array}$ \\
\hline
\end{tabular}

Saleh et al.: Peripheral (Seventh) Nerve Palsy and Multiple Sclerosis: A Diagnostic Dilemma - A Case Report

12 Bassetti C, Bogousslavsky J, Barth A, Regli F: Isolated infarcts of the pons. Neurology 1996;46:165-175.

13 Baugh RF, Basura GJ, Ishii LE, Schwartz SR, Drumheller CM, Burkholder R, et al: Clinical practice guideline: Bell's palsy. Otolaryngol Head Neck Surg 2013;149(3 suppl):S1-S27.

14 Misawa S, Kuwabara S, Mori M, Hayakawa S, Sawai S, Hattori T: Peripheral nerve demyelination in multiple sclerosis. Clin Neurophysiol 2008;119:1829-1833.

15 Uzawa A, Mori M, Ito S, Kuwabara S: Neurological picture. Isolated abducens and facial nerve palsies due to a facial collicular plaque in multiple sclerosis. J Neurol Neurosurg Psychiatry 2011;82:85-86.

$\$ 16$ Agarwal R, Manandhar L, Saluja P, Grandhi B: Pontine stroke presenting as isolated facial nerve palsy mimicking Bell's palsy: a case report. J Med Case Rep 2011;5:287.

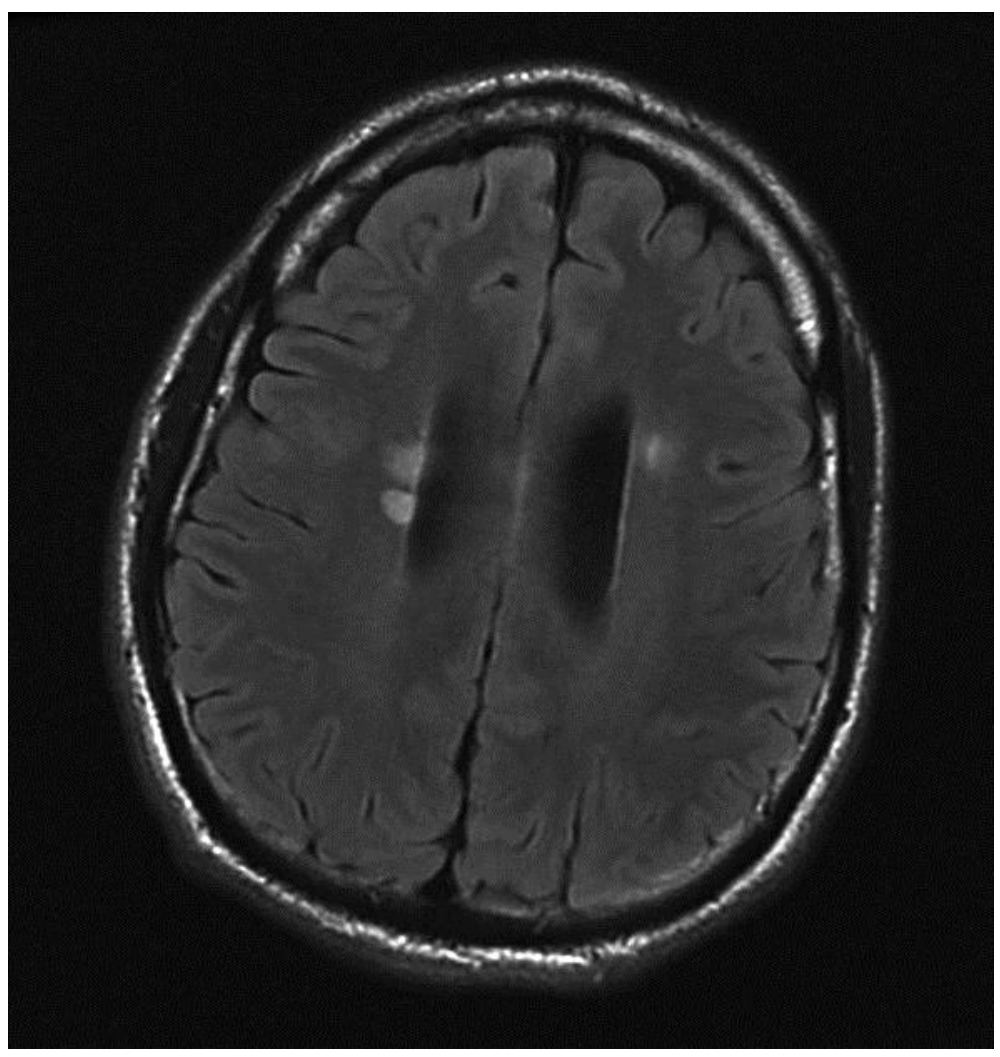

Fig. 1. MRI imaging showing two right-sided periventricular lesions. 
Saleh et al.: Peripheral (Seventh) Nerve Palsy and Multiple Sclerosis: A Diagnostic Dilemma - A Case Report

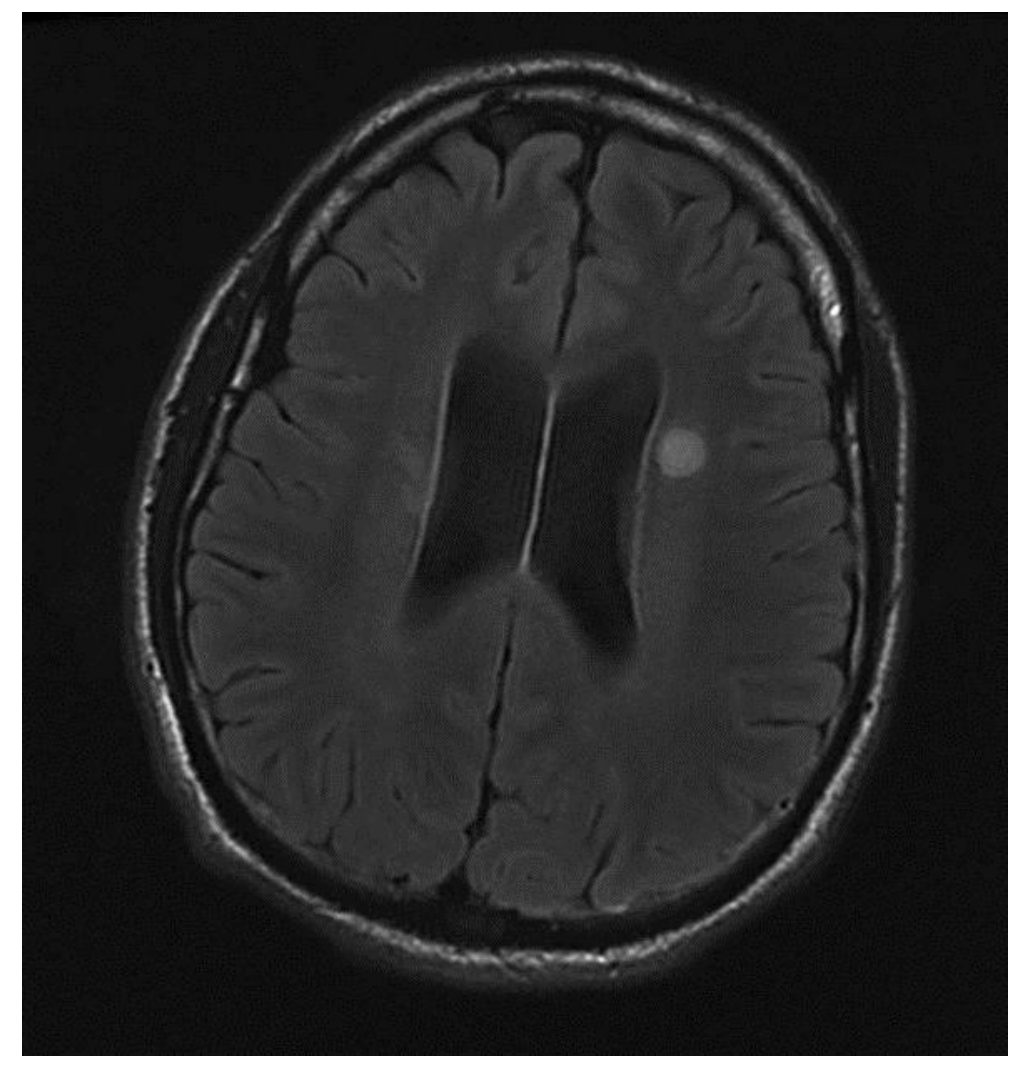

Fig. 2. MRI imaging showing one left-sided periventricular lesion. 
Saleh et al:: Peripheral (Seventh) Nerve Palsy and Multiple Sclerosis: A Diagnostic Dilemma - A Case Report

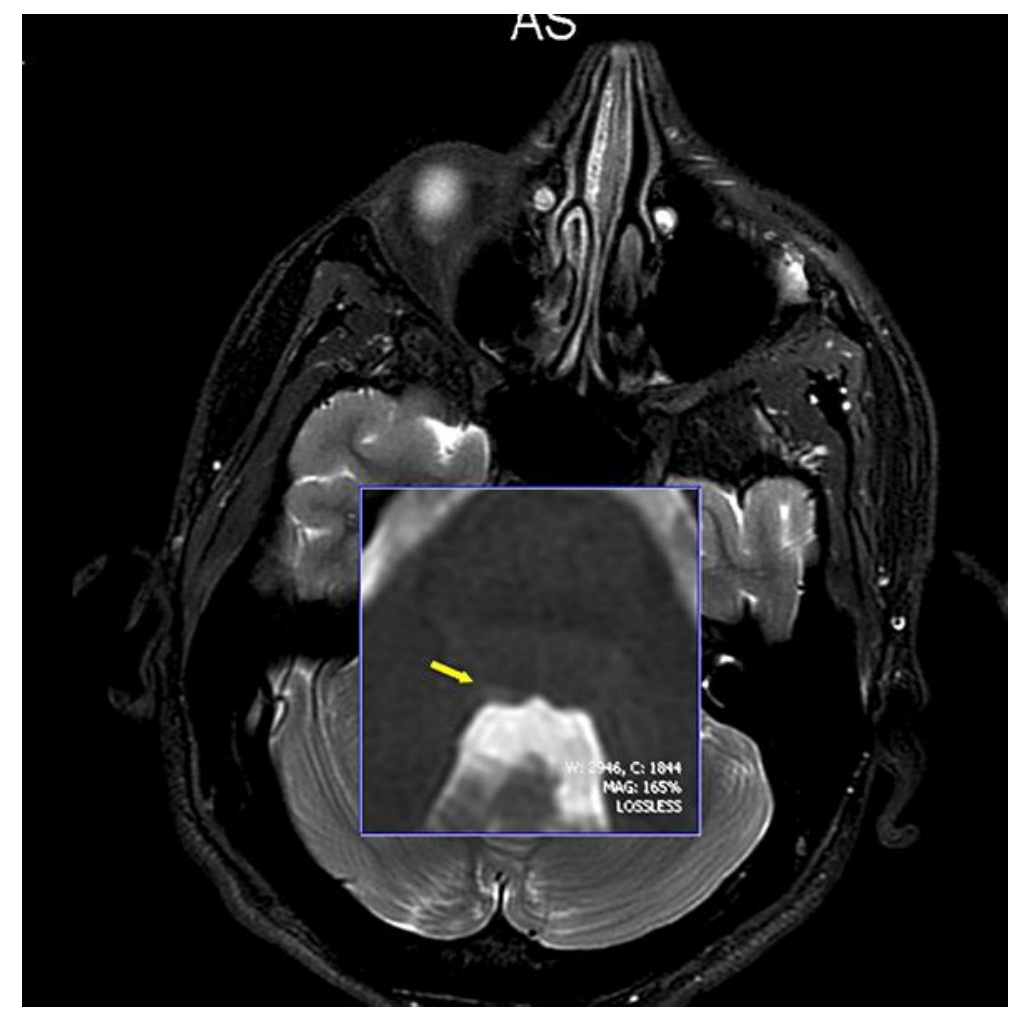

Fig. 3. MRI of the spine with a tiny right-sided pontine lesion (yellow arrow). 